\title{
IDENTITÄTSPROBLEME VON TÜRKEN IN DER DEUTSCHEN GESELLSCHAFT $^{1}$
}

\author{
Dr. phil. Mustafa ACAR \\ Forschungszentrum für Türkei-EU-Deutschland (Zf-TEUD) \\ (Türkiye-Avrupa Birliği-Almanya Araştırmalar Merkezi)
}

\begin{abstract}
ZUSAMMENFASSUNG
Die Identitätsprobleme und Identitätskonflikte von Ausländern sind in Deutschland seit langer Zeit ein soziologisches Untersuchungsthema. Dieses Thema wird nach dem jeden Anschlag, der in Europa im Namen des Islams verwirklicht wird, auf allen Ebenen immer wieder diskutiert und wird mit den Migrations- und Integrationsthemen verbunden. In diesem Zusammenhang werden besonders die religiösen, nationalen und kulturellen Identitäten von muslimischen Migranten, die als Integrationshindernisse angesehen werden, debattiert und diese Gruppe wird oft kritisiert. Die Türken sind wegen ihrer deutlichen Mehrheit die erste Adresse dieser Kritik. Nach dem Anschlag in Frankreich hat die anti-islamische Haltung von PEGIDA, die tausende Menschen auf der Straße sammeln kann, auf die Wiederverschärfung der Diskussion um die Identität von Muslimen gewirkt. Die zweiten und dritten Generationen von Türken, die in zwei unterschiedlichen Kulturen leben, betonen angesichts dieser Kritik ihre religiösen Identitäten, die von den Werten ihrer nationalen und kulturellen Identitäten unterstützt werden. Diese Haltung wird von der Mehrheitsgesellschaft, die im Allgemeinen die Integration als die Assimilation definiert, als Integrationsdefizite angesehen. Das Ziel dieser Untersuchung besteht darin, den Prozess der Identitätsentwicklung von türkischen Jugendlichen, die wichtigen Identitätsarten in diesem Prozess und die als Identitätskrise angesehenen Kulturkonflikte und Desintegrationsthemen zu debattieren.

Schlüsselwörter: Identität, Identitätsprobleme, Identitätsarten, Desintegration, Kultureller Konflikt.
\end{abstract}

\section{ALMAN TOPLUMUNDA TÜRKLERIN KIMLIIK SORUNLARI}

\section{ÖZ}

Sosyolojik olarak Almanya'da uzun zamandan bu yana bir araştırma konusu olan yabancıların kimlik sorunları ve .çatışması, Avrupa'da Islam'a addedilerek gerçekleştirilen her olumsuz eylemden sonra her düzeyde yeniden tartışmaya açılarak

${ }^{1}$ Makalenin geliş tarihi: 04.02.2015

Makalenin kabul tarihi: 11.03.2015 
göç ve uyum konuları ile ilişkilendirilmektedir. Bu bağlamda özellikle müslüman kökenli göçmenlerin dini, kültürel ve ulusal kimlikleri tartışlmakta ve bunların uyuma engel olduğu tezi üzerinden hareketle bu göçmen grubu siklıkla eleştirilmektedir. Almanya'da sayısal üstünlüğünden dolayı Türkler bu eleştirinin ilk muhatabı olmaktadırlar. Fransa'daki saldırıdan sonra, Almanya'da müslümanların kimlik tartışmalarının yeniden alevlenmesine on binleri sokağa döken PEGIDA'nın anti-islam tutumu da etkili olmuştur. Hem Alman hem de Türk kültürü içinde yaşayan ikinci ve üçüncü kuşak Türkler, bu eleştiriler karşısında özellikle dini kimliklerini öne çıkarmakta ve bunu ulusal ve kültürel kimlik değerleri ile desteklemektedirler. Bu tutum, иуити genellikle asimilasyon üzerinden tanımlayan baskın toplum tarafindan bir uyum eksikliği olarak görülmektedir. Bu çalışma, Türk gençlerinin kimlik geliştirme süreci, bu süreçte öne çıkan kimlik türleri, kimlik bunalımı olarak görülen kültürel çatışma ve uyumsuzluk konularını tartışmayı amaçlamaktadır.

Anahtar Kelimeler: Kimlik, Kimlik Sorunlarl, Kimlik Türleri, Uyumsuzluk, Kültürel Çatışma.

\section{EINLEITUNG}

Identität, Identitätsbildung und Identitätskonflikt sind jederzeit als wichtige Begriffe zu betrachten. In individuellen und kollektiven Ebenen gibt es viele Identitätsmöglichkeiten. Ein Individuum entwickelt sich innerhalb seiner Umgebung, die überwiegend seine soziale, kulturelle und religiöse Identität formt. Wenn sich die soziale oder geographische Umgebung und andere viele Faktoren des Individuums, z. B. durch Migration, verändern, beeinflussen diese neuen Faktoren die Herausbildung der Identität des Individuums. Besonders in der heutigen globalisierten und internationalisierten Zeit fällt es in europäischen Gesellschaften auf, dass Individuen und Minderheitsgruppen in den Mehrheitsgesellschaften in einem Spannungsfeld des kulturellen Spektrums die Konflikte leben.

Obwohl es sich heutzutage bei der ersten Generation von Türken in der deutschen Gesellschaft um die kulturellen, religiösen oder individuellen Identitätsprobleme nicht mehr handelt, ist eine Expansion der sozialwissenschaftlichen Veröffentlichungen zum Thema „kulturelle, religiöse, nationale, politische und hybride Identitäten" der zweiten und dritten Generationen von Türken besonders unter der allgemeinen Forschung „Migration und Identität“ zu beobachten. Diese beschäftigen sich eigentlich überwiegend mit der Problematik der Migration. Die Diskussion um die Identität von Migranten wird besonders mit den aktuellen politischen und gesellschaftlichen Themen (Selefisten, radikaler Islam und der Aufstieg des Anti-Islamismus in Europa) thematisiert. Obgleich besonders die Religion von Migranten jahrzehntelang keine Rolle gespielt hat, werden heute der Islam, die Muslime und deren religiöse Identität in Deutschland wieder debattiert.

Die Radikalisierung des Islams durch die unterschiedlichen radikalislamischen Terrororganisationen wie ISIS (Islamischer Staat im Irak und in Syrien), Al-Nusra-Front und Islamische Front in Syrien und dem Irak 
beeinflussen besonders die jungen Muslime in Deutschland. Nach der Erklärung des Innenministers Thomas de Maizière gibt es im Irak und in Syrien deutlich mehr ISIS-Kämpfer aus Deutschland: „Wir gehen von 550 aus, bis vor wenigen Tagen haben wir noch 450 gesagt" (Bildzeitung, 2014). ${ }^{2}$ Die schnelle Ausbreitung dieser Organisationen unter den Jugendlichen in Deutschland hat eine Gegenwirkung in der deutschen Gesellschaft und verursacht die Erhöhung des Einflusses des antimuslimischen und ausländerfeindlichen Vereins „Patriotische Europäer gegen die Islamisierung des Abendlandes“ (PEGIDA). PEGIDA organisiert seit 20. Oktober 2014 wöchentliche Demonstrationen besonders in Dresden und in anderen deutschen Städten. Obgleich PEGIDA in ihrer ersten Demonstration nur „350 Teilnehmer“ erreicht hat, hat sich die Zahl der Demonstranten nach einem Monat auf „17.500“ (Polizei Sachsen, 2014) ${ }^{3}$ gesteigert. In diesem Zusammenhang konzentriert sich die Diskussion in Deutschland auf die Identitätsprobleme der zweiten und dritten Generationen von muslimischen Migranten. In dieser Diskussion haben die Türken eine besondere Bedeutung, weil sie die größte Gruppe von Migranten in Deutschland bilden. Deshalb sind die Deutschlandtürken im Vordergrund, wenn es um eine Islamdebatte geht.

Die zweiten und dritten Generationen der türkische Migranten sind überwiegend in Deutschland geboren und aufgewachsen. Sie werden in der deutschen Gesellschaft sozialisiert und leben in zwei unterschiedlichen Kulturen. Aufgrund dieser Situation „,neigen sie zu Veränderungen ihrer sozialen Identitäten“ (Polat, 1997: 14). Es gibt zahlreiche Untersuchungen über die Begriffe Identitätskonflikte und Identitätsprobleme, die die türkische Jugendliche in Deutschland thematisieren. Diese Thematisierung der Identität der zweiten und dritten Generationen von Türken bezieht sich im Allgemeinen auf die unterschiedlichen und widersprüchlichen kulturellen Erwartungen oder Anforderungen der türkischen Gesellschaft und Aufnahmegesellschaft. In diesem Zusammenhang stellen sich die Fragen: Was ist eigentlich „Identität“ und welche Erscheinungsformen sind für die türkischen Jugendlichen wichtig? Haben die türkischen Jugendlichen Identitätskonflikte? Welche Einflüsse hat der Migrationsprozess auf die Identitätsbildung? Unter diesen Fragen ist es das Ziel der vorliegenden Untersuchung, ihre Identitätsbildung und die Identitätsprobleme der zweiten und dritten Generationen von Türken in Deutschland $\mathrm{zu}$ analysieren. Im Rahmen der empirischen Analysen dieser Arbeit werden die Daten des Stiftungszentrums für Türkei Studien und des

\footnotetext{
${ }^{2}$ Unter: http://www.bild.de/politik/inland/thomas-de-maiziere/550-isis-kaempfer-indeutschland38675226.bild.html, abgerufen: 03.12.2014.

${ }^{3}$ Unter: http://www.polizei.sachsen.de/de/MI_2014_33591.htm, abgerufen: 05.01.2015.
} 
Migrations- und Politikforschungszentrums der Hacettepe Universität (HUGO) genutzt.

\section{DER BEGRIFF „IDENTITÄT“}

Die Identität ist ein stark umstrittener Begriff und die Verwendung in allen Bereichen der Sozialwissenschaften ist nicht mehr klar und einheitlich. Dieses gilt für alle Arten von Identität wie persönliche oder soziale Identität, religiöse oder nationale Identität. Wegen der steigenden Untersuchungen, Veröffentlichungen, Vorstellungen und Definitionen von Autoren und Wissenschaftlern zur Themen "Identität“ und "Identitätsarten“ ist die Diskussion noch komplexer geworden. In diesem Zusammenhang hat Erikson (1970: 11-12) lange zu betont, dass die Begriffe „Identität" und „Identitätskrise“ noch mehr ritualistisch verwendet werden. Das Gleiche gilt auch noch heutzutage.

Die Identität ist zuerst ein Entwicklungsprozess und bildet sich im Verlauf der Individuation heraus. „Identität kann als eine relativ stabile Einheit, Gleichheit und Kontinuität des Individuums in einer Gesellschaft, oder als die Kompetenz eines Individuums verstanden werden“ (Öktem/Öktem, 1985: 76). Die Definitionen der Autoren implizieren unterschiedliche Richtungen des Begriffs. Castells (2002: 8) definiert die Identität als ,, die Quelle von Sinn und Erfahrung für die Menschen“. Andererseits wird Identität als ein Prozess betont. Nach Meyer (2002: 41) ist die Identität ,ein offener Prozess des Aushandelns zwischen dem Selbstbild, das der Einzelne von sich entwirft, und dem Bild, das sich seine sozialen Handlungspartner in wechselnden Zusammenhängen von ihm machen." Die Identitätstheorie des symbolischen Interaktionismus geht von dem Konzept der Rollenübernahme aus. „Vereinfacht könnte man zunächst sagen: Identität seien meine Vorstellungen von dem Bild, das andere von mir haben“ (Heckmann, 1993: 196). Das Drei-Faktoren Modell spricht über die drei Identitäten: „Soziale Identität bedeutet ein komplexes Gebilde von Attitüden. Personelle Identität bedeutet die einzigartige Kombination von Daten der Lebensgeschichte; sie meint das Fremd- und Selbstwahrnehmen. Ich-Identität bedeutet das subjektive Empfinden der eigenen Situation, Kontinuität und Eigenart, die ein Individuum allmählich als ein Resultat seiner verschiedenen sozialen Erfahrungen erwirbt" (ebd., 196).

In diesem Zusammenhang ist soziale Identität die Summe von kultureller Identität und Rollenidentität. Mit der Rollenidentität wird „die Erwartungen der Gesellschaft an den Inhaber/die Inhaberin einer bestimmten Position, so z.B. die Position der Lehrerin und die Rolle als Lehrerin“ (Öktem/Öktem, 1985: 77) gemeint. Soziale Identität ist der Sinn, den eine Person für sich schafft und sich an deren Werten und Merkmalen orientiert und die von der Person internalisiert werden. Die Person benutzt diese Werte und Merkmale, um sein Leben zu gestallten. Eine Person richtet ihr Leben an Kriterien aus, die der Person helfen, in einer sozialen Gemeinschaft zu leben. Es 
geht hier auch um religiöse oder individualistische Merkmale. Die Menschen leben in einer Gemeinschaft mit ähnlichen Kriterien und an denen sie sich orientieren. Sie bilden anhand der Ausrichtung dieser Kriterien ihre Identität aus. Man versteht unter der Bildung sozialer Identität „einen Prozess der Sinnkonstruktion auf der Grundlage eines kulturellen Attributes oder einer entsprechenden Reihe von kulturellen Attributen, denen gegenüber andere Quellen von Sinn Priorität zugesprochen wird“" (Meyer, 2002: 41). Sowohl die personale Identitätsbildung als auch die soziale Identitätsbildung ist ein komplexer und schwieriger Prozess. Identität ist „,die Quelle von Spannung und Widerspruch“" (ebd., 41).

Wenn es sich bei dem Migrationsprozess in einer Einwanderungsgesellschaft um die Identitätsauseinandersetzungen handelt, entstehen drei wichtigen Begriffe im Zusammenhang der Identitätsprobleme: Kultur, Nationalität und Religion. Weil diese drei Begriffe wichtige Rolle bei der Bildung der Identität einer Person spielen. Neben diesen Begriffen wird die hybride Identität, die die kulturellen Räume der Menschen erweitert, stark diskutiert. In diesem Sinne sollen diese Begriffe kurz erläutert werden.

\section{A. Kultur und Identität}

Der Begriff „Kultur“ wird in den Sozialwissenschaften vielseitig verwendet und es gibt zahlreiche Definitionen und Kontroversen. Im Allgemeinen bezieht sich der Kulturbegriff auf die Vereinheitlichung aller Tätigkeiten und Ausdrucksformen einer Gesellschaft oder eines Volkes und einer Nation. Im Zusammenhang mit dem Gegenstand hat die Kultur wegen des interkulturellen Verständnisses in Einwanderungsgesellschaften vor allem als „Orientierungssystem“ (vgl. Auernheimer, 2003: 74) Bedeutung. In diesem Zusammenhang hat die Kultur für das Individuum einen herausragenden Stellenwert und wird als Kultur sowohl als ein System von Werten und Normen, als auch als Verhaltenserwartungen verstanden. Andererseits wird die Kultur von Auernheimer (2003: 74) als die ,symbolische Seite der sozialen Praxis" angesehen.

Das Individuum orientiert sich in seiner sozialen Umwelt und handelt gemäß den kulturell Adäquaten dieser Umwelt. Diese Situation ist vom kulturellen Wissen der Bedeutung von Handlungen und von der Kenntnis der kulturellen Werte und Normen der Gesellschaft abhängig. Die Kultur hat einen großen Einfluss auf das Alltagsleben der Menschen, was sich auf diese Weise auch in den vorherrschenden Verhältnisformen und Institutionen wiederspiegelt. „Eine Kultur enthält die Landkarten der Bedeutung, welche die Dinge für ihre Mitglieder verstehbar machen. Die Landkarten der Bedeutung trägt man nicht einfach im Kopf mit sich herum: sie sind in den Formen der gesellschaftichen Organisationen und Beziehungen objektiviert, durch die ein Individuum zu einem gesellschaftlichen Individuum wird. Kultur ist die Art, wie die sozialen Beziehungen einer Gruppe strukturiert und geformt sind; aber sie ist auch die 
Art wie diese Formen erfahren, verstanden und interpretiert" (Clarke u.a., 1981: 41).

Über die Themen Kultur und Identität gibt es verschieden Meinungen. Neben den vielen kritischen Arbeiten auch von Migranten selbst, die die traditionellen Identitäts- und Kulturkonzepte in Frage stellen (Kalpaka/Räthzel, 1986-1994; Atabay, 1994; Otyakmaz, 1995; Prasad, 1993), wird von der Mehrheit die ethnische Kultur und das Identitätsverständnis angenommen. Dieses Verständnis sieht Kultur und Identität als die nationale Zugehörigkeit bzw. bei den Generationen die Nationalität und Herkunftskultur ihrer Familien an. Insbesondere die türkischen Migranten der ersten Generation leben in der mitgebrachten Kultur weiter und akzeptieren viele Veränderungen nicht. Ethnische Zugehörigkeit und die weiterlebende Tradition in der Migration werden als Widerstand gegen die Integration angesehen. Demgegenüber spricht man nicht über die migrationspezifischen Veränderungen.

Nach den zahlreichen Studien zur Identifikation mit der Herkunftskultur (Bukow, 1999; Hämmig, 2000; Weidacher, 2000; Tietze, 2001; Badawia/Hamburger/Hummrich, 2003; Sackmann/Schultz/Prümm/Peters, 2005; Reiff, 2006; Öztoprak, 2007; Hans, 2010) wird die kulturelle Identität bei den zweiten und dritten Generationen über die Eltern vermittelt und auch durch Fremdzuschreibung verstärkt. Andererseits „muss die Identifikation mit der Herkunftskultur nicht direkt mit dem Wunsch nach Abgrenzung von der Aufnahmegesellschaft, deren Werte in erster Linie durch die außerfamiliären Sozialisationsinstanzen vermittelt werden, verbunden sein" (vgl. Sauer, 2014:88). Es ist auch möglich, dass sich die Menschen mit Migrationshintergrund mit den Kulturen der Aufnahmegesellschaft und eigenen Gesellschaft identifizieren oder eine neue kulturelle Identifikation (Mischidentität oder bikulturelle Identität) entwickeln können.

Die partiellen Vorstellungen von Kultur und unvollständige Wahrnehmung von Migranten dienen lediglich der Verfestigung von Klischeebildern über Migranten, die mittlerweile schon seit Jahrzehnten hier leben. „Bei einer solchen Herangehensweise bleibt wenig Raum für Migranten, denn die Tatsache, dass ihr Alltag aus mindestens zwei Kulturelementen besteht, wird nicht wahrgenommen. So entsteht ein gewisser Konservatismus, der die Existenz neuerer kultureller Formen gerne ignoriert. Dieser Ignoranz fallen auch die neuen kulturellen Ausdrucksformen zum Opfer, die im Laufe der verschiedenen Migrationsgeschichten entstanden sind und die der Realität vor allem jüngerer Migranten mehr entsprechen als herkömmliche akzeptierte Kulturen"(Prasad, 1993: 63).

\section{B. Nationale Identität und Migration}

Bindung an eine ethnische Gruppe ist ein Teil der sozialen Identität eines jeden Menschen. Die Ich- Identität hilft dem Einzelnen in einer Minderheitsgruppe, die Erwartungen und Informationen zu filtern bzw. sie in 
Handlungen umzusetzen (vgl. Heckmann, 1992: 198). Kulturelle Identität ist „,das Kollektive im Individuum. Durch die Internalisierung kultureller Normen werden Individuen Angehörige einer Gesellschaft. Kulturelle Identität ist somit eine ethnische Identität. Im Rahmen der bestehenden bürgerlichen Nationalstaaten bedeutet ethnische Identität zumeist gleichermaßen nationale Identität. Dabei ist die Religion, als Ensemble tradierter Werturteile und der Weltanschauung, ein Aspekt der Kultur" (Öktem/Öktem, 1985: 77). Nach Elwert (1982: 721) ist „Selbstbewusstsein immer auch kulturelle Identität“".

Identitätsbildung ist besonders schwierig in der Migrationsbewegung. Die Migranten leben im Aufnahmeland mit einer anderen Nationalität. Zwischen den Migranten und Mitglieder der Aufnahmegesellschaft gibt es „symbolische Grenzen, die über die Staatszugehörigkeit gezogen und durch den Pass repräsentiert werden" (Penitsch, 2003: 16). Mitglieder der Aufnahmegesellschaft erhalten aufgrund ihrer Zugehörigkeit zur Dominanzgesellschaft bei der Geburt den deutschen Pass, der ihnen Privilegien und Handlungsrechte in Deutschland und in Europa zusichert. Durch den Besitz des deutschen Passes werden ihnen die Rechte (Wahlrecht usw.) gewährt, ohne dass sie etwas dafür tun müssen. Nationale Identität ist mit der Staatszugehörigkeit verbunden. Jeder Mensch hat eine Nationalität. Diese Wahrnehmung hängt von einer Nation bestimmender Begriffe ab. „Die Normen und Werte, die einen Nationalstaat definieren, haben die Funktion, soziale Kohäsion unter den Nationalstaatszugehörigen zu schaffen" (ebd. 16). Nationale Solidarität schafft die emotionale Bindung an der Nation. „Von jeder Person wird erwartet, sich zur eigenen Gruppe zu bekennen, ihre nationale Identität als wichtig zu erachten“ (ebd., 16). Jeder Nationalstaat verlangt von den Menschen, die in seiner Grenzen leben, emotionale Bindung. Auch in der Bundesrepublik Deutschland wird die nationale Identiät durch die Einheit von Staatsvolk, Nationalkultur und Staatsgebiet gekennzeichnet (vgl. Heckmann, 1992: 211). In der Konzeption der deutschen Staatsbürgerschaft gibt es „ein Identitätsprogramm, das die politische Zugehörigkeit zur Gesellschaft vornehmlich an ethnische Kriterien knüpft" (Tietze, 2001: 188). Das ist ein zweiteiliger Prozess, der im alltäglichen Leben (Erfahrungen und Orientierungen) ,von unten“ und als Perspektive „,von oben“ durchgeführt wird (vgl. Jaschke, 2001: 179f.).

Wenn man akzeptiert, dass kulturelle Identität die nationale Identität ist, wird diese Identität und die ethnische Grenze zwischen der türkischen Bevölkerung und der Aufnahmegesellschaft von den in Deutschland lebenden Türken stark wahrgenommen. Die Situation der türkischen Jugendlichen in Deutschland ist eine ganz andere als die ihrer Eltern. Sie sind zum größten Teil in Deutschland geboren oder zumindest aufgewachsen. Nach Goldberg, Halm und Sauer (2001:108). „kennzeichnet die Kinder der Gastarbeiter eine Bikulturalität, verortet zwischen dem neuen Heimatland Deutschland und der 
eigenen Herkunft, die sich in der Familie, den sozialen Netzwerken und nicht zuletzt der Sprache als mehr oder weniger dauerhaft erwiest"

Die Migranten der zweiten Generation erleben im stärkeren Masse Kultur- und Identiätskrisen und befinden sich in einem -vielfach belastendenBalanceakt bzw. Loyalitätskonflikt zwischen den Werten und Anforderungen der Kultur ihrer Eltern und Großeltern (vgl. Weische, 1982; Weber, 1989). Die meistens türkischen Familien versuchen zunehmend, dem drohenden Verlust der nationalen, kulturellen und religiösen Identität vor allem durch ein rigideres Festhalten an traditionellen Normen $\mathrm{zu}$ begegnen. Andererseits wird die kulturelle Identität der Türken in der deutschen Umgebung nicht anerkannt, aber geduldet. In diesem Sinne betonen Goldberg, Halm und Sauer (2001: 41), dass „sich insgesamt sowohl bei Deutschen als auch bei Türken die größten Differenzen im ethnisch- kulturellen Bereich zeigen“ und dass "sich beide Gruppen hier mehrheitlich anders sehen." Die Angst vor Identitätsverlust und das aufgrund der Negativhaltung des Umfeldes erzeugte Bedürfnisse, das Selbstwertgefühl zu halten, führen zu Strategien der ethnischen Isolation. Nach Uzun (1993: 59) „hat diese bei der türkischen Minderheit in Deutschland zwei Versionen, die extrem nationalistische und die religiös-fundamentalistische."

Nach El-Mafaalanı und Toprak (2011: 50-51) kann man seit den1990er Jahren deutlich beobachten, dass die türkischen Jugendlichen, die in Deutschland geboren und aufgewachsen sind, ihre türkische Identität selbstbewusster und selbstverständlicher nach außen präsentieren. Die Gründe für das verstärkte Betonen der türkischen Identität können in vier Punkten konkretisiert werden: Die ausländerfeindlichen Übergriffe auf türkische Migranten in den 1990er Jahren haben das Gefühl des Zusammenhalts innerhalb der Gemeinde verstärkt. Die Eltern und ihre Kinder fühlen sich durch den Zuzug von Spätaussiedlern benachteiligt. Aus Sicht der Eltern bekommen die Spätaussiedler mehr Rechte, obwohl sie - im Gegensatz zu ihren Kindern kaum Deutsch können. Als weiteren Grund für den Rückzug in die türkische Identität kann das Scheitern der beruflichen und sozialen Integration in die hiesige Gesellschaft genannt werden. Und es sind die Erfolge türkischer Sportler, zum Beispiel im Fußball, mit denen sich die türkischen Jugendlichen intensiv identifizieren.

\section{Religiöse Identität}

Neben den kulturellen und nationalen Identitäten ist die religiöse Identität eine wichtige Variation der kollektiven Identität. Religiöse Identität hat mehrere Dimensionen, die die Verwendung des Begriffs verbreiten. Wenn es sich bei den Diskussionen um die Religion und Identität handelt, wird die Religion im Allgemeinen als Wurzel der persönlichen und sozialen Identität, als die Grundlage der Nationalidentität und Gruppenzugehörigkeit und als der Sicherung der Normen und Traditionen angesehen. 
Religiöse Identität wird von den Forschungsrichtungen immer unterschiedlich definiert. Während der Begriff sozialpolitisch als ein Element der staatlichen Stabilität verstanden wird und vom Staat unterstützt werden muss, wird er ethnografisch als ein Faktor für die Entstehung von Völkern und Kulturen beschrieben. In diesem Sinne versteht man religiöse Identität als ein bestimmtes Element und bedeutender Faktor der nationalen Identität. Dagegen wird religiöse Identität soziologisch als Stabilisator der gesellschaftlichen Prozesse und als Basis für Moral und Traditionen wahrgenommen. Obwohl die Philosophie die religiöse Identität als eine philosophische Kategorie sieht, ihre Inhalte erforscht und immanente Widersprüche ihrer individuellen sowie sozialen Bestandteile diskutiert, wird der Begriff theologisch als die Bestimmung der Religionszugehörigkeit verstanden und als bindendes Element der Religionsgemeinden angesehen. Religiöse Identität bezieht sich im Kontext der Globalisierung auf den Dialog zwischen unterschiedlichen Kulturen (vgl. Krylov, 2012: 260).

Alle diese Dimensionen der religiösen Identität haben eine besondere Bedeutung für die Menschen im Migrationsprozess. In diesem Sinne ist die Erhaltung und Fortsetzung der religiösen Identität für die Deutschland-Türken besonders wichtig, weil die türkischen Familien vor dem kulturellen, nationalen und besonders islamischen Identitätsverlust ihrer Kindern immer Angst haben. Aufgrund dieser Angst haben die deutschen Schulen keine Bedeutung für sie. „Ein allgemeiner Religionsunterricht, wie er in deutschen Schulen angeboten wird, reicht türkischen Eltern nicht aus. Für die sunnitischen Eltern geht es in erster Linie um das Vermitteln der islamischen Pflichten. Weil diese Unterweisung in der Schule nicht gewährleistet wird, erfahren die Koranschulen, die meistens auch unter Kulturvereinen geführt werden“" (ElMafaalanı/Toprak, 2011: 51). Obwohl der Islam in Deutschland wegen der Türken ,die zweigrößte Religionsgemeinschaft“ darstellt und ,sich seit der 90er Jahren eine eigene religiöse Infrastruktur gebildet hat" (Şen/Aydın, 2002: 7), haben die türkischen Eltern die Angst vor der religiösen Entfremdung ihrer Kinder nicht verloren.

Die Islam-Debatte in Deutschland wird oft über die zweite und die dritte Generationen durchgeführt. Im Gegensatz zur allgemeinen Vorstellungen „bleibt Religion wichtig sowohl für die zweite wie auch teilweise dritte Generation der vorwiegen türkischen Muslime und Musliminnen in Deutschland. Gewandelt hat sich der Zugang zu sowie der Umgang mit religiösen Werten und Ritualen. Wird der ersten Generation noch ein emotionaler und als selbstverständlich gelebter eher wenig reflektierter Glaube attestiert, ist der Zugang der Religion der meist schon in Deutschland aufgewachsenen Kinder ein anderer: Er muss unteranderem in einer nichtislamischen Umwelt den Kindern durch die Eltern erklärt, aber auch der Mehrheitsgesellschaft plausibel gemacht werden, was einem kognitiven eher 
rationalem Umgang mit religiösen Glaubensformen und Geboten förderlich ist “ (Kaweh, 2006: 1). Die Hinwendung der zweiten und dritten Generationen von Muslimen zu islamischen Werten und Normen wird im Allgemeinen als ,ein Prozess der Selbstkonstitution als Moralsubjekt und Selbstbehauptung“ (Nökel, 1999: 124) angesehen.

Bei der Bildung der religiösen Identität (gleichzeitig auch der kulturellen und nationalen Identität) von Folgegenerationen im Migrationsprozess sind zwei wichtige Einflüsse im Vordergrund: Familie und Herkunftscommunity. Für die Migranten der zweiten und dritten Generation ist die Familie nicht nur der Ort, wo sie Zugang zu ihrer Herkunftskultur, Verwandtschaft und ein Stück Heimat finden, sondern auch ein Ort, wo sich religiöse, kulturelle und nationale Identität der Folgegenerationen bildet und auslebt. Mit der Auswirkung der zunehmenden Moscheen verstärkt man eine türkische Binnenkultur, in der man versucht, das traditionelle Leben weiterzuführen. Aber in einem fremden Land und einer fremden Kultur ist der Schutz der traditionellen Verhaltensweisen nur bedingt möglich. Darum werden die Auseinandersetzungen über die türkische Kultur und deren Lebensformen von den Türken als eine Bedrohung wahrgenommen. „Je massiver die Bedrohung ist, desto intensiver versucht man im Kernbereich des traditionellen Zusammenhalts zu wahren und zu verstärken, um ihn damit zu schützen“ (Straube, 1987: 44).

Die Bildung der Communities ist eine Folgeerscheinung der Migration. Sie ist ein internationales Phänomen und eine weitverbreitete Handlungsweise, in jedem Land, wo Migranten leben, ein Stück Heimat und Sicherheit zu erhalten. Für die meisten Migranten der ersten Generation nimmt die Herkunftscommunity einen wichtigen sozialen Wert ein und wird zu einer wichtigen Bezugsgruppe, weil sie Geborgenheit, soziale Kontakte, Selbstbewusstsein und Unterstützung in den verschiedensten Lebenslagen bietet. Diese ethnischen Communities als eine neue Form des kollektiven Handels haben materielle wie auch ideelle Funktionen für ihre Mitglieder. Herkunft-Community ist auch eine Kommunikationsmöglichkeit, um aus der gesellschaftlichen Isolation als Migranten in einem fremden Land herauszukommen und bietet zugleich einen Raum, wo die migrationsspezifischen Erfahrungen Gehör finden. Zugleich bietet die Community kollektive Identitätsentwürfe, die aus Teilen der mitgebrachten Herkunftskultur und aus neu erworbenen kulturellen Formen in der Migration bestehen.

Die einzelnen Mitglieder der Gemeinschaft stehen in einem Abhängigkeitsverhältnis zueinander. Neben all den emotionalen und sozialen Versorgungen der einzelnen Mitglieder kann die Community aber auch eine Kontrollinstanz darstellen (vgl. Lutz, 1991: 142). Die Mitglieder der Community fühlen sich verpflichtet, dem Normen-, Religions- und 
Wertesystem, dem Kohäsionsdruck ihrer Community nachzukommen, um die Erhaltung ihrer gemeinschaftlichen Integrität und die Sicherheiten, die ihnen diese Community bietet, weiter zu bewahren. Neben dem Herkunft-Community „suchen junge Türken ihre Wurzeln zunehmend im Islam. Weitgehend unbemerkt von der deutschen Öffentlichkeit ist seitdem eine islamische Kultur mit religiösen Vereinigungen, islamischen Bestattungsinstituten und Modegeschäften entstanden“"(Spiewak, 1999: 34-35). Das ermöglicht, die Jungen in derCommunity zu halten.

\section{Hybride Identität}

Der Begriff „Hybridität“ wurde eigentlich im 19. Jahrhundert aus der postkolonialen Theoriebildung entwickelt. „Hybride Identität“ hatte eine negative Bedeutung und ihre Träger wurden für die menschlichen Rassen als eine Gefahr angesehen (vgl. Hein, 2006: 54). Hybride Identität wird heute meistens mit den Wörtern wie multikulturell, interkulturell, zweiheimisch, binational usw. zusammen gebraucht und ist mit den Menschen mit Migrationshintergrund eng verbunden (vgl. Badawia, 2002 und Mecheril, 2004). In diesem Zusammenhang ,,bedeutet hybride Identität, dass ein Mensch sich zwei oder mehreren kulturellen Räumen gleichermaßen zugehörig fühlt. Die Untersuchung konzentriert sich auf jene Individuen, die einen muslimischen Migrationshintergrund haben und diesen mit einer nationalen Identität verbinden“ (Foroutan/Schäfer, 2009: 11). Dieser Begriff identifiziert sich in der Öffentlichkeit meistens mit den Ausländern. Die Hybriddefinition von Hein im Bezug auf kulturelle Identität ist noch breiter und umfassender: „Die Kategorie des Hybriden wird schließlich auch in Bezug auf kulturelle Identitäten diskutiert. Dabei bezieht sich der Begriff hybrider Identitäten auf die Erfahrung zwischen den Kulturen. Hybride Identitäten bezeichnen Identitäten, die in der Auseinandersetzung, Verbindung und Kreuzung unterschiedlicher kultureller Kontexte entstehen. Hybride Identitäten sind kulturelle Identitäten im Übergang " (Hein, 2006 434).

Im Zusammenhang der Migration wurde die hybride Identität von Stuart Hall thematisiert. Nach Hall steht in einer modernen Einwanderungsgesellschaft auf der einen Seite die Moderne (Wir) und auf der anderen Seite die Tradition (das Andere). In einer solchen Gesellschaft werden die Identitätsbildungen durchgeschnitten. In einem fremden Land haben die Migranten miteinander enge und starke Verbindungen. Sie wollen ihre Herkunftskultur nicht verlieren und sich nicht assimilieren. Deshalb pflegen sie ihre Kultur und Traditionen ununterbrochen. Andererseits haben sie mit anderen kulturellen Systemen auch Verhältnisse. Es handelt sich bei diesem neuen kulturellen System um eine neue Artikulation und Sozialisation. In diesem Prozess entwickelt sich hybride Identität. Die hybride Identitätsbildung hängt hier von der Stärke der ethnischen, kulturellen und religiösen Identifikation ab, weil „die kulturelle Hybridbildung zur Untergrabung nach innen gerichteter 
Kulturkonzepte beiträgt, die dem romantischen Nationalismus, dem Rassismus, dem Ethnozentrismus, der religiösen Rivalität, dem Kulturchauvinismus und dem kulturellen Essentialismus zugrunde liegen“ (Aydin, 2003: 68).

Im Migrationsprozess setzt sich die hybride Identitätstheorie zum Ziel, dass sich die Migranten nicht in einer „Entweder-Oder" Situation, sondern in „Sowohl-als-auch“ Situationen befinden. Wegen der wachsenden Zahl von muslimischem Migranten in Deutschland werden die großen Differenzen zwischen der Mehrheitsgesellschaftskultur und der Kulturen von Migranten und die Entfremdungsprozesse beider Seiten als eine Bedrohung des gesellschaftlichen Friedens angesehen. In einer solchen gesellschaftlichen Situation benötigt Vermittler und gegenseitigen Kommunikationskanäle auf beiden Seiten. In diesem Zusammenhang wird die Vermittlerrolle von den Trägern hybrider Identitäten erwartet. „,Träger hybrider Identitäten können letztendlich auch als „Brückenmenschen“ oder Mediatoren auf der internationalen Ebene fungieren. Sie spielen eine bedeutende Rolle im Annäherungsprozess zwischen Europa und den Herkunftsländern der muslimischen Migrantinnen und Migranten. [...] In Netzwerken von Migranten mit muslimischem Hintergrund, die sich zwischen Europa, der Türkei, Nordafrika und dem Nahen Osten bewegen, bilden sich zunehmend hybride Identitäten aus. Diese können als Träger konstruktiver Identitätsangebote im Sinne einer vertieften politischen und kulturellen Partizipation in den europäischen Mehrheitsgesellschaften fungieren " (Foroutan/Schäfer, 2009: 18). Obwohl es zahlreiche hybride Migranten mit muslimischen Hintergrund, die sich voll integriert haben, gibt, lebt die überwiegende Mehrheit von Migranten in Europa und Deutschland ihre eigene Kulture weiter. Es gibt nicht nur einen Entfremdungsprozess zwischen den Kulturen der Auf- und Ausnahmegesellschaften, sondern auch zwischen den hybriden und traditionalen Migranten.

\section{IDENTITÄTSPROBLEME VON TÜRKISCHEN} JUGENDLICHEN

Die Identitätsbildung der zweiten und dritten türkischen Generationen wird in Deutschland immer als problematisch angesehen. Diese Problematik ist eigentlich zweiseitig: Die Identitätsbildung der türkischen Jugendlichen sind sowohl von türkischer Seite (von den Integrationsdefizite), als auch von der deutschen Seite (von restriktiven Partizipationsmöglichkeiten) abhängig. Die Grundlage des türkisch-seitigen Problems ist der so genannte Kulturkonflikt zwischen familiären Traditionen der Herkunftskultur und modernen Lebenskultur der Aufnahmegesellschaft. „Eine Identität zwischen aufnehmender und Herkunftskultur herzustellen, ist das entscheidende Kriterium, um die jungen Ausländer der nicht bloß neben den beiden Kulturen aufwachsen $\mathrm{zu}$ lassen und in doppelter Halbsprachigkeit in eine Orientierungslosigkeit, d.h. in unzureichende Chancen eigner umfassender 
Identitätsbildung zu entlassen“ (Reich, 2008: 165). Nach Auernheimer (1988: 9) „war die Doppelstrategie zwischen Integration ins Aufnahmeland und Erhaltung der kulturellen Identität des Herkunftslandes gescheitert.“

Obwohl man in der deutschen Gesellschaft nur eine nationale oder kulturelle Identität, „entweder Deutsch oder Türke“, erwartet und diese Positionierung klar sein muss, enthält diese Position gleichzeitig eine Abgrenzung zur Aufnahmegesellschaft, weil diese Positionierung von Migranten als die Identitätsassimilierung verstanden wird. Dagegen wird die Beibehaltung der Herkunftskultur von der Aufnahmegesellschaft als die nicht gelungene Integration verstanden. In diesem Zusammenhang beziehen sich die Probleme der Identitätsbildung von türkischen Jugendlichen in Deutschland auf die Integrationsprobleme. Im Zusammenhang der Identitätsbildung stehen drei Probleme im Vordergrund, wenn es um die türkische Nachfolgegenerationen geht: Die geringere Schul- und Ausbildung, das Leben in einer Parallelgesellschaft und die Identifikationsprobleme der zweiten und dritten türkischen Generationen. Außerdem wird die steigende Religiosität als eine Gefahr für die Identifikation mit Deutschland angesehen, weil „der Gebrauch des Begriffs Islam in einigen Diskurssträngen nahelegt, dass dieser von der Mehrheitsgesellschaft als etwas sozusagen Gegenteiliges zum Deutschen wahrgenommen wird" (Spielhaus, 2006: 30).

Schulische Bildung und berufliche Ausbildung sind wichtige Faktoren für die Integration der zweiten und dritten Generationen, weil die Schüler dort einen großen Teil ihrer Zeit verbringen. Vor allem spielen die Schulen für die türkischen Kinder und Jugendlichen eine wichtige Rolle, um Deutsch zu lernen, mit gleichaltrigen Deutschen in Kontakt zu kommen, ein neues Umfeld und neue Perspektiven kennen zu lernen. Die berufliche Ausbildung und die Eingliederung in das Beschäftigungssystem bieten nicht nur die Gewähr für einen sicheren Lebensunterhalt, sondern auch durch die betrieblichen Strukturen Möglichkeiten zur Zusammenarbeit mit Menschen anderer Herkunft. Andererseits sind die Schulbildung und Ausbildung die Basis für die Einbindung in den Arbeitsmarkt. Ohne Schulbildung ist die berufliche Ausbildung nicht möglich und ohne berufliche Ausbildung ist die Arbeitschance eingeschränkt. 40,9\% der türkischen Männer und 60,3\% der türkischen Frauen haben keine berufliche Ausbildung (vgl. Sauer 2014: 26). Obwohl die türkische Folgegenerationen in Deutschland geboren und aufgewachsen sind, „weisen sie weiterhin deutlich geringere Bildungsbeteiligungsquoten auf als sonstige Migranten" (Autorengruppe Bildungsbericht, 2012: 7). Als Grund für die geringe Schulbildung der Nachfolgegeneration wird ,häufig die Bildungsferne und der geringe soziale Status der Eltern genannt, die ihr insgesamt geringes, im Ausland erworbenes Bildungsniveau an ihre Kinder ,vererben“, denn die soziale Herkunft bedingt in Deutschland in hohem Maß die Schulbildungserfolge - auch in der 
Mehrheitsgesellschaft" (Sauer, 2014: 23). Es ist die allgemeine Annahme, dass ohne die Teilhabe am deutschen Bildungssystem national-kulturelle Identität von türkischen Jugendlichen verstärkt wird, weil das geringe Bildungsniveau einer der Gründe der sozialstrukturellen Marginalisierung ist.

Im Allgemeinen glaubt man in Deutschland, dass die Türken eine Parallelgesellschaft gebildet haben. „Mit Parallelgesellschaften wird in der öffentlichen Debatte die Vorstellung von ethnisch homogenen Bevölkerungsgruppen verbunden, die sich räumlich, sozial und kulturell von der Mehrheitsgesellschaft abschotten. Der Begriff impliziert zugleich massive Kritik an der Lebensweise von Migrantinnen und Migranten und enthält die Forderung nach kultureller Assimilation" (Belwe, 2006: 2). Obgleich Parallelgesellschaften als Integrationshindernis und als Widerspruch gegen die ideale Gesellschaft angesehen wird, „wird dabei nicht berücksichtigt, dass Rückzug auch eine Reaktion auf Abgrenzungen durch die Mehrheitsgesellschaft, mangelnde Teilhabemöglichkeiten und Hindernisse der Aufnahmegesellschaft sein kann" (Sauer, 2009: 150). Nach der Definition von Thomas Meyer (2002: 343ff.) gibt es fünf Indikatoren für die Existenz von Parallelgesellschaften: kulturell-religiöse Homogenität, lebensweltliche und zivilgesellschaftliche Segregation, Verdopplung der mehrheitsgesellschaftlichen Institutionen, formal freiwillige Segregation und siedlungsräumliche Segregation. Die Wohnverhältnissen, interkulturellen Kontakten, Diskriminierungserfahrungen und ethnischen Infrastrukturen von türkischen Migranten werden seit 1999 von der Stiftung Zentrum für Türkeistudien repräsentativ befragt. Diese Daten werden gemäß der von Meyer entwickelten Indikatoren für Parallelgesellschaften durch die Merkmale Religiosität (kulturelle Homogenität), Kontakte zu Deutschen (lebensweltliche Segregation), Organisationsgrade (Verdopplung von Institutionen), Diskriminierung (Freiwilligkeit von Segregation) und ethnische Quartiersbildung (Wohnraumsegregation) operationalisiert. Nach den Ergebnissen ,ist die zunehmende Religiosität das einzige Merkmal einer Parallelgesellschaft, das im Untersuchungszeitraum tatsächlich linear in Richtung der Entwicklung parallelgesellschaftlicher Strukturen weist. Alle anderen Merkmale deuten jedoch nicht auf das Anwachsen parallelgesellschaftlicher Strukturen hin" (Sauer, 2009: 151).

In diesem Sinne hat die religiöse Parallelgesellschaft eine große Bedeutung für die Identitätsbildung von türkischen Jugendlichen, weil sich die klare Tendenz auszeichnet, sich von nationaler Identität in religiöse Identität zu verwandeln. „Im öffentlichen Diskurs um die (Des-)Integration von Zuwanderern weicht die früher übliche nationale Zuweisung als Ausländer oder Türke zunehmend der religiös-kulturellen Zuweisung Muslime“ (Sauer, 2012: 39). Nach einer Studie, die im Jahr 2013 vom Migrations- und Politikforschungszentrum der Hacettepe Universität (HUGO) und vom Institut 
für Markt- und Meinungsforschung Data $4 \mathrm{U}$ durchgeführt wurde, identifizieren sich $37,72 \%$ der befragten türkischen Einwanderer als „türkische Muslime”, während 13,57\% sich als „Türken“ identifizieren, 13,97\% als „türkischmuslimische Deutsche“ und 8,08\% als „türkische Deutsche“. ${ }^{4}$ Die islamische Zugehörigkeit wird als Integrationsdefizit und die Ursache der Parallelgesellschaften angesehen und die nationale Herkunft, Kultur und Religion werden in der Wahrnehmung häufig vermischt (vgl. Schiffauer, 2008: 15). In einem Vergleich nach Jahren fällt eine deutliche Zunahme der Religiosität auf. Als Ursache dieser Zunahme ist anzunehmen, dass „die Diskussion seit dem 11. September 2001 um das Wesen des Islams die Identifikation mit dem Islam“ verstärkt hat und dass die Menschen Angst „,vor einem Identitäts- und Werteverlust im Zuge der fortdauernden Migration“ (Sauer 2012: 40) haben. Zwischen 2003 und 2010 lag der Anteil der sehr und eher Religiösen mit geringen Schwankungen um 75\%, 2011 steigt er jedoch erneut auf nunmehr $82 \%$. Der Anteil der sehr und eher Religiösen beträgt im Jahr 2012 80,7\% und im Jahr 2013 82\% (vgl. Sauer, 2012: 40 und Sauer, 2014: 143).

Ein anderes Diskussionsthema um die Identität ist die Identifikation von türkischen Jugendlichen mit Deutschland und mit der deutschen Gesellschaft. Das „Sich-Deutsch-fühlen“ bei den türkischen zweiten und dritten Generationen wird von der deutschen Gesellschaft meistens als wichtige und erfolgreiche Integration bewertet. Dagegen wird die Beibehaltung der Herkunftsidentität als gescheiterte Integration verstanden, weil die Assimilation im öffentlichen Diskurs als gelungene Integration gesehen wird. In diesem Zusammenhang wird die Verbundenheit mit dem Herkunftsland und der Herkunftskultur vom Identifikationsverständnis der Mehrheitsgesellschaft abgelehnt. „In zahlreichen Studien zur kulturellen Identität wurde nachgewiesen, dass die Identifikation mit der Herkunftskultur, die bei den nachfolgenden Generationen insbesondere über das Elternhaus vermittelt wird, aber auch durch Fremdzuschreibung gefestigt werden kann“" (Sauer, 2014: 88). Natürlich ist es auch möglich, dass sich ein Individuum mit beiden Kulturen identifiziert oder eine bikulturelle Identität entwickelt.

Nach den Ergebnissen der Studie des Stiftungszentrums für Türkeistudien zeigen sich die Unterschiede in dem Verbundenheitsgefühl nach Geschlecht und Zuwanderungsgeneration. 37,4\% der Frauen und 49,1\% von Männern fühlen sich mit der Türkei verbunden. Dagegen identifizieren sich 18,3\% von Männern und 21,7\% der Frauen mit Deutschland. Der Anteil der Identifikation beider Länder liegt bei den Frauen bei 33\% und bei den Männern bei $27,6 \%$. Die Generationen zeigen im Zusammenhang mit der

4 Vgl. http://dtj-online.de/tuerken-fuehlen-sich-in-deutschland-wohl-13871, abgerufen: 29.01.2015. 
Heimatsverbundenheit interessante Verteilungen auf: $34,7 \%$ der ersten Generation, 45\% der zweiten Generation, 45\% der dritten Generation und 49\% von Heiratsmigranten identifizieren sich mit der Türkei. Demgegenüber fühlen sich $21,2 \%$ der ersten Generation, $21 \%$ der zweiten Generation und 25\% der dritten Generation mit Deutschland. 37,1\% der ersten Generation, 30,9\% der zweiten Generation und 20\% der dritten Generation sehen sowohl Deutschland als auch die Türkei als ihre Heimat an. 10\% der dritten Generation sieht sich keinem Land verbunden. Dieser Anteil liegt bei der ersten Generation bei 7,1\% und bei der zweiten Generation bei 3,1\%. Es fällt hier auf, dass sich die dritte Generation noch häufiger bikulturell definiert und dass die Identifikation von den zweiten und dritten Generationen mit der Türkei noch höher als die erste Generation liegt. Diese Untersuchung zeigt auch auf, dass die Identifikation weniger Einfluss auf das Einbürgerungsverhalten von Türken hat. Hier stehen rechtliche (Gleichstellung, Wahlrecht, Aufenthaltssicherheit) und pragmatische Gründe (Vermeidung von bürokratischem Ärger, Reisefreiheit) im Vordergrund (vgl. Sauer, 2014: 89-114).

Die wichtigste Grundlage des Problems der deutschen Seite sind die gesetzlichen Beschränkungen, die die Identitätsentwicklungen der türkischen Jugendliche verhindern. Die rechtlichen Rahmenbedingungen der Partizipation sind in Deutschland restriktiv. Demokratische Partizipationsmöglichkeiten in allen wichtigen Bereichen werden aufgrund der Staatsbürgerschaft eingeschränkt. In diesem Sinne wird die Integration auch unter dem Aspekt der staatsbürgerlichen Rechte und der damit verbundenen politischen Partizipation verstanden. Diese Menschen ohne deutsche Staatsbürgerschaft durften in Deutschland seit Jahrzehnten weder wählen noch gewählt werden. Die Migranten können ihre politischen Rechte nur gewährleisten, wenn sie die deutsche Staatsbürgerschaft erwerben. Die Einbürgerung ist der einzige Weg, um gleichberechtigt an Wahlen und an der politischen Willensbildung teilzuhaben. Ohne deutsche Staatsbürgerschaft ist die Beteiligung am politischen Willensbildungsprozess begrenzt, weil die Migranten nicht zum Wahlvolk gehören und somit in der Regel von der zentralen demokratischen Mitbestimmungsform ausgeschlossen sind. In Deutschland wird die nationale Identität als die Konstruktionsregel für die Migranten angesehen. Deshalb werden das Staatsbürgerschaftsrecht, die Einbürgerung, die politischen und sozialen Partizipationsmöglichkeiten und die Möglichkeit des Zusammenlebens durch diese Regel bestimmt (vgl. Heckmann, 1992: 211).

\section{SCHLUSSFOLGERUNG}

Anhand der oberen Bestimmungen zeigt sich offensichtlich, was für ein komplexer Prozess die Identitätsbildung von türkischen Jugendlichen ist. Migration ist auch im soziokulturellen Kontext ein Prozess und hat einen großen Einfluss auf die Identitätskonstruktion der betroffenen Personen. Migranten, die in ihrem Heimatland eine kulturelle, nationale und religiöse 
Identität haben, überschreiten nationalstaatliche Grenzen und leben in einem anderen Land mit einer anderen Gesellschaft, die auch eigene nationale, kulturelle und religiöse Identität haben. Es wurde festgestellt, dass Migranten in der Mehrheitsgesellschaft ihre Kultur, Nationalität und Religion mehr als die Menschen im eigenen Land pflegen und betonen, obwohl sie voll integriert sind. Somit entwickelt sich ein Wir-Gefühl in der Fremde. Es ist ein schmerzhafter Prozess, eine neue Identität in einem solchen Fall zu entwickeln, weil die betroffenen Personen sowohl die Erwartungen von der Mehrheitsgesellschaft, als auch von eigener Gesellschaft berücksichtigen müssen.

Einerseits stellt sich die Frage nach ihrer Integration in das politische und soziale System der Aufnahmegesellschaft besonders dringlich. Andererseits lässt sich argumentieren, dass die Türken aus einem anderen Kulturkreis kommen, dass sie in Deutschland durch die Moscheen, türkische Läden, Kindergärten usw. eine Parallelgesellschaft entwickeln und dass eine zunehmende Einbeziehung in die deutsche Gesellschaft gar nicht mehr möglich ist. Trotz dieser Argumentation wird einerseits Deutschland in hohem Maß (78\%) der Türken als Zuhause empfunden, zugleich bleibt aber die Verbundenheit mit der Türkei bestehen. Andererseits sehen sich zugleich auch $73 \%$ als sehr anders als Deutsche und nur 42\% stehen ihnen sehr nah. Während sich mehr als die Hälfte hin- und hergerissen zwischen den beiden Ländern sieht, wollen $49,1 \%$ von Türken zugleich die deutsche und die türkische Lebensweise einfach zusammenbringen und sehen in der Bikulturalität keinen Konflikt. Dagegen fühlt sich nur knapp ein Drittel als marginalisiert, also heimatlos und nirgends zugehörig (vgl. Sauer, 2014: 92). Der radikalisierende Teil der Jugendlichen im Zusammenhang der Nationalität und Religiosität entsteht aus diesen Marginalen. Die negativen Ereignisse durch diese marginalen Radikalen stehen in der deutschen öffentlichen Meinung und den Medien meistens mit dem Islam in Verbindung und werden mit der Migration, Integration und den Identitätskonflikten von Migranten zusammen diskutiert. Nach Ahmad Mansour sind die Jugendlichen, die sich radikalisieren, nach Syrien und in den Irak reisen und für den Heiligen Krieg kämpfen, auf der Suche nach Sinn und nach Halt. Und bei diesen Gruppierungen finden sie Sicherheit, Zufriedenheit und eine Mission. Viele Jugendliche, die anfällig für diese Gruppierungen sind, suchen nach einer Vaterfigur. Sie brauchen Freunde und eine Umwelt, in der sie sich wohlfühlen. Und das finden sie bei diesen Gruppierungen (vgl. Mansour, 2014) ${ }^{5}$

Das Ergebnis der vorliegenden Arbeit zeigt, dass es keine einzige Identität der zweiten und dritten Generationen von Türken in Deutschland gibt

5 Unter: http://www.rbb-online.de/politik/beitrag/2014/09/ahmad-mansour-isislamismus-berlin.html, abgerufen: 02.02.2015. 
und dass sie sich in einer Komplexität der Identitätsbildung befinden. In diesem Zusammenhang soll der Begriff Identität im Migrationsprozess nicht als ein Problem, das gelöst werden muss, angesehen werden. Wenn die unterschiedlichen Identitätsentwicklungen der Migranten als ein Problem bezeichnet werden, bleibt diese Thema für immer problematisch, weil die Identitätsbildung ein offener Prozess ist. Die türkischen Migranten der zweiten und dritten Generationen müssen alltäglich mit verschiedenen Wertvorstellungen leben und sich der jeweiligen Umgebung anpassen. Diese Situation beeinflusst natürlich auch ihre Persönlichkeitsentwicklung. Obwohl die hybride Identität als eine Lösung in Betracht gezogen wird, handelt sich auch bei den hybriden Identitäten um die Identitätsdiffusion oder Identitätskonflikte. In diesem Sinne werden die türkischen Jugendlichen meistens als eine Generation ohne Ziele bezeichnet. In einer Demokratie auf der globalisierten Welt darf jeder Mensch ohne Druck seine Identität im Rahmen der demokratischen Wertvorstellungen entwickeln und leben. Einige Menschen können beide Kulturen als gewinnbringende wertvolle Ressourcen ansehen, während sich die Anderen als Türke, Deutsche oder Muslime definieren. In diesem Sinne benötigt ein neues multikulturelles Gesellschaftverständnis.

\section{LITERATUR}

AUERNHEIMER, Georg (2003), Einführung in die interkulturelle Erziehung, (3. Aufl.), Wiss. Buchges., Darmstadt.

AUERNHEIMER, Georg (1988), Der sogenannte Kulturkonflikt. Orientierungsprobleme ausländischer Jugendlicher, Campus Verlag, Frankfurt a.M., New York.

ATABAY, Ilhami (1994) Ist dies mein Land? Identitätsentwicklung türkischer Migrantenkinder und jugendlicher in der Bundesrepublik, Centaurus Verlag, Herbolzheim.

AUTORENGRUPPE BILDUNGSBERICHT (2012), Bildung in Deutschland 2012, http://www. bildungsbericht.de/daten2012/bb_2012.pdf, abgerufen: 28.01.2015.

AYDIN, Yasar (2003), Zum Begriff der Hybridität, Sozialökonomischer Text $\mathrm{Nr}$. 105 , https://www.wiso.unihamburg.de/fileadmin/sozialoekonomie/fachbereich/_dwp ordner/Forschung/Publikationen/Zum_Begriff_der_Hybriditt.pdf, abgerufen: 15.01.2015.

BADAWIA, Tarik/HAMBURGER, Franz/HUMMRICH, Merle (2003), Wider die Ethnisierung einer Generation. Beiträge zur qualitativen Migrationsforschung, Frankfurt a. M./London.

BADAWIA, Tarik (2002), Der Dritte Stuhl - Eine Grounded TheoryStudie zum kreativen Umgang bildungserfolgreicher Immigrantenjugendlicher mit kultureller Differenz, IKO-Verlag, Frankfurt/M. 
BELWE, Katharina (2006), „Parallelgesellschaften: Editorial“, Aus Politik und Zeitgeschichte, Nr., 1-2/2006, S. 2:

BUKOW, Wolf Dietrich (1999), „Ethnisierung der Lebensführung“, Migration und Traditionsbildung, (Hrsg.) Apitzsch, Ursula, Wiesbaden, S. 92104.

CASTELLS, Manuel (2002), Die Informationsgesellschaft II: Die Macht der Identität, Leske- Budrich, Opladen.

CLARK，John/HALL，Stuart/JEFFERSON, Tony/ROBERTS，Brian (1981), „Subkulturen, Kulturen, Klasse“, Jugendkultur als Widerstand: Milieus, Rituale, Provokationen, (Hrsg.), Honneth, Axel/ Linder Rolf/ Paris, Rainer, Frankfurt a.M., S. 39-135.

EL-MAFAALANI, Aladin/TOPRAK, Ahmet (2011), Muslimische Kinder und Jugendliche in Deutschland: Lebenswelten - Denkmuster Herausforderungen, Konrad-Adenauer-Stiftung e.V, Druckerei Franz Paffenholz GmbH, Bornheim.

ELWERT, Georg (1982), „Probleme der Ausländerintegration. Gesellschaftliche Integration durch Binnenintegration?", Kölner Zeitschrift für Soziologie und Sozialpsychologie 34, S. 717-731

ERIKSON, Erik (1970), Jugend und Krise. Die Psychodynamik im sozialen Wandel, Klett, Stuttgart.

GOLDBERG, Andreas/HALM, Dirk/SAUER, Martina (2002), Migrationsbericht 2002 des Zentrums für Türkeistudien, LIT Verlag, Münster.

FOROUTAN, Naika/SCHÄFER, Isabel (2009), „Hybride Identitäten muslimische Migrantinnen und Migranten in Deutschland und Europa“, Aus Politik und Zeitgeschichte, Nr., 5/2009, S. 11-18. Hamburg.

HALL, Stuart (1994), Rassismus und kulturelle Identität, Argument,

HÄMMIG, Oliver (2000), Zwischen zwei Kulturen. Spannungen, Konflikte und ihre Bewältigung bei der zweiten Ausländergeneration, Springer Verlag, Opladen.

HANS, Silke (2010), Assimilation Oder Segregation? Anpassungsprozesse von Einwanderern in Deutschland, Springer Verlag, Wiesbaden.

HECKMANN, Friedrich (1992), Ethnische Minderheiten, Volk und Nation: Soziologie innerethnischer Beziehungen, Enke Verlag, Stuttgart.

HEIN, Kerstin (2006), Hybride Identitäten. Bastelbiografien im Spannungsverhältnis zwischen Lateinamerika und Europa, Transcript Verlag, Bielefeld.

JASCHKE, Hans-Gerd (2001), Rechtsextremismus und Fremdenfeindlichkeit: Begriffe, Positionen, Praxisfelder, VS Verlag für Sozialwissenschaften, Wiesbaden. 
KALPAKA, Annita/RÄTHZEL, Nora (1994), Die Schwierigkeit, nicht rassistisch zu sein, DIJ Verlag, Köln.

KALPAKA, Annita/RÄTHZEL, Nora (1986), Handlungsfähigkeit statt „Integration“, DJI Verlag, München.

Deutschland,

KAWEH, Silvia (2006), Religion und Identität junger Muslime in rs.de/interreligioserdialag/061213_Kaweh_jungemuslime.pdf, abgerufen: 14.01.2015.

KRYLOV, Alexander (2012), Religiöse Identität: Individuelles und kollektives Selbstbewusstsein im postindustriellen Raum, West-Ost-Verlag, Berlin und Verlag Ikar, Moskau.

LUTZ, Helma (1991), Welten Verbinden. Türkische Sozialarbeiterinnen in den Niederländen und in der Bundesrepublik Deutschland, IKO Verlag, Frankfurt a. M.

MECHERIL, Paul (2004), „Andere Deutsche gibt es nicht. Zusammenhänge zwischen subalterner Erfahrung und diskursiver Praxis", The Black Book. Deutschlands Häutungen, (Hrsg.), AntiDiskriminierungsBüro Köln und cyberNomads, Frankfurt/M, S. 82-90.

MEYER, Thomas (2002), Identitätspolitik. Vom Missbrauch kultureller Unterschiede, Suhrkamp, Frankfurt am Main.

MEYER, Thomas (2002): „Parallelgesellschaften und Demokratie“, Die Bürgergesellschaft. Perspektiven für Bürgerbeteiligung und Bürgerkommunikation, (Hrsg.) Meyer, Thomas/Weil, Reinhard, Verlag Dietz, Bonn, S.343-372.

NÖKEL, Sigrid (1999), „Islam und Selbstbehauptung Alltagsweltliche Strategien junger Frauen in Deutschland“, Der neue Islam der Frauen. Leibliche Lebenspraxis in der globalisierten Moderne- Fallstudien aus Afrika, Asien und Europa, (Hrsg.), Hessling, Ruth/Nökel, Sigrid/Werner, Karin, Transcript Verlag, Bielefeld.

OTYAKMAZ, Berrin Ö. (1995), Auf allen Stühlen, Das Selbstverständnis junger türkischer Migrantinnen in Deutschland, Köln.

ÖKTEM, Ayşe/ÖKTEM, Özcan (1985), „Kulturelle Identität, Sozialisation und Sprache bei türkische Arbeiterkinder in der Bundes Republik Deutschland“, Interkulturelle Kommunikation, (Hrsg.), Jochen Rehbein, Günter Narr Verlag, Tübingen, S. 70-102.

ÖZTOPRAK, Ümit (2007), Identitäts- und Akkulturationsstile türkischer Jugendlicher, Frankfurt:

PENITSCH, Regine (2002), Migration und Identität, Weissensee Verlag,Berlin.

POLAT, Ülger (1997), Soziale und kulturelle Identität türkischer Migranten der zweiten Generation, Verlag Kovac, Hamburg. 
PRASAD, Nivedita (1993), Feministische Sozialarbeit mit Immigrantinnen, Diplomarbeit, Freie Universität- Berlin.

REICH, Kersten (2008), „Demokratie im Konzept der Interkulturellen Pädagogik bei Georg Auernheimer: vom so genannten Kulturkonflikt zur pluralen Interkulturellen Pädagogik“, Macht - Kultur - Bildung, (Hrsg) Lisa Rosen, Schahrzad Farrokhzad, WAXMANN Verlag, Münster/New York/ München/Berlin, S.165-176

REIFF, Gesa (2006), Identitätskonstruktionen in Deutschland lebender Türken der 2. Generation, Ibidem Verlag, Stuttgart.

SACKMANN, Rosemarie/ SCHULTZ, Tanjev/PRÜMM, Kathrin/PETERS, Bernhard (2005), Kollektive Identitäten: Selbstverortungen türkischer MigrantInnen und ihrer Kinder, Peter Lang Verlag, Frankfurt am Main.

SAUER, Martina (2014), Integrationsprozesse, wirtschaftliche Lage und Zufriedenheit türkeistämmiger Zuwanderer in Nordrhein-Westfalen, Ergebnisse der Mehrthemenbefragung 2013, eine Analyse in Kooperation mit dem Ministerium für Arbeit, Integration und Soziales des Landes NordrheinWestfalen, $\quad$ http://www.tamvakfi.de/downloads/bericht-nrw2013_end.pdf, abgerufen: 12.01.2015.

SAUER, Martina (2012) Integrationsprozesse türkeistämmiger Migrantinnen und Migranten in Nordrhein-Westfalen, Ergebnisse der zwölften Mehrthemenbefragung, Eine Analyse in Kooperation mit dem Ministerium für Arbeit, Integration und Soziales des Landes NordrheinWestfalen, http://www. zfti.de//downloads/nrw-bericht-2011_end.pdf, abgerufen: 28.01.2015.

SAUER, Martina (2009), Teilhabe und Orientierungen Türkeistämmiger Migrantinnen und Migranten in NRW, Ergebnisse der zehnten Mehrthemenbefragung, eine Analyse im Auftrag des Ministeriums für Arbeit, Integration und Soziales des Landes Nordrhein Westfalen, http://www.zfti.de//downloads/ downmehrthemenbefragung2009.pdf, abgerufen: 28.01.2015.

SCHIFFAUER, Werner (2008); Parallelgesellschaften. Wie viel Wertekonsens braucht unsere Gesellschaft? Für eine kluge Politik der Differenz, Transcript Verlag, Bielefeld.

SPIELHAUS, Riem (2006), „Religion und Identität: Vom deutschen Versuch, „Ausländer“ zu „Muslimen“ zu machen“, IP-DIE ZEITSCHRIFT, Ausgabe März 2006, S. 28-36.

SPIEWAK, Martin (1999), „Neue Heimat Islam“, Zeitpunkte, Nr. 2/99,

STRAUBE, Hanne (1987), Türkisches Leben in der Bundesrepublik Deutschland, Campus Verlag,

Frankfurt - New York 
UZUN, Ertuğrul (1993), „Gastarbeiter, Immigranten, Minderheit. Vom Identitätswandel der Türken in Deutschland“, Deutsche Türken. Das Ende der Geduld, (Hrsg.), Claus Leggewie/Zafer Şenocak, Rowohlt Taschenbuch Verlag, Reinbek bei Hamburg.

ŞEN, Faruk/AYDIN, Hayrettin (2002), Islam in Deutschland, Verlag C.H.Beck, München.

TIETZE, Nikola (2001), Islamische Identitäten. Formen muslimischer Religiosität junger Männer in Deutschland und Frankreich, Hamburger Edition, Hamburg.

WEBER, Cora (1989), Selbstkonzept, Identität und Integration, VWB für Wissenschaft und Bildung, Berlin.

WEIDACHER, Alois (2000), In Deutschland zu Hause. Politische Orientierungen griechischer, italienischer, türkischer und deutscher junger Erwachsener im Vergleich - DJI-Ausländersurvey, Opladen.

WEISCHE, Alexa (1982), Sozial- kulturelle Probleme Junger Türken in der Bundesrepublik Deutschland, Köln. 